\title{
ON A GENERALIZED DIRICHLET PROBLEM FOR PLURI- SUBHARMONIC FUNCTIONS AND PSEUDO-CONVEX DOMAINS. CHARACTERIZATION OF ŠILOV BOUNDARIES
}

\author{
BY \\ H. J. BREMERMANN( $\left.{ }^{1}\right)$
}

1. Introduction. To continuous real boundary values prescribed on the boundary of a domain $D$ in the complex number space $C^{n}, n>1$, there does not exist, in general, a pluriharmonic function (i.e. real part of a holomorphic function) that assumes the given boundary values.

For $n>1$ the real parts of holomorphic functions (satisfying $\partial^{2} h^{\prime} / \partial z_{\mu} \partial \bar{z}_{\nu}=0$ for $\mu, \nu=1, \cdots, n)$ are a proper subclass of and do no longer coincide with the harmonic functions (satisfying $\sum_{\mu=1}^{n} \partial^{2} h / \partial z_{\mu} \partial \bar{z}_{\mu}=0$ ). The harmonic functions solve the Dirichlet problem uniquely but they are not invariant under holomorphic transformations. The latter property, however, seems to be quite desirable and is indispensable if the theory is to be extended to complex manifolds.

S. Bergmann $[4 ; 5 ; 6]$ has been the first to suggest not to give up at this point but to extend the class of pluriharmonic functions such that the boundary value problem becomes uniquely solvable, the functions are pseudoconformally invariant, and as many properties of the pluriharmonic functions are preserved as possible (compare \$2). His method while having certain advantages is limited to a special class of domains.

We suggest in the following a different method that applies to all domains of holomorphy with a "smooth" boundary and can be considered as the direct extension of the Perron-Carathéodory method (Perron [29], Carathéodory [15]) from one to several complex variables. In this method, given a domain $D$ in the plane of one complex variable, one takes the subharmonic functions in $\bar{D}$ that are less than or equal to the prescribed values on the boundary $\partial D$ of $D$. It is easy to show that the upper envelope of this class of functions is harmonic, and if the boundary of $D$ is "sufficiently nice", this upper envelope function assumes exactly the given boundary values, and hence the Dirichlet problem is solved.

The analog for several complex variables is elaborated in this paper. The subharmonic functions (of one complex variable) are replaced by the plurisubharmonic functions which constitute their proper generalization to several

Received by the editors June 20, 1957 .

(1) This research was supported by the United States Air Force, through the Office of Scientific Research. 
variables. One big difference that arises: in general the upper envelope is not pluriharmonic, but only plurisubharmonic.

Also while for complex dimension one (and sufficiently nice boundaries) we may prescribe arbitrary boundary values on the whole boundary this is no longer true for higher dimension. Only for strictly pseudo-convex domains the values are prescribed on the whole boundary, while for instance in the case of analytic polyhedra the values are prescribed only on a subset of real dimension $n$ of the topological boundary.

The treatment of general pseudo-convex domains requires the notion of "Silov boundary" (see §6), a notion which so far has played a role in Banach algebras (Mirkil [23], Arens and Singer [1]). It is shown (Theorem 7.2): Let $D$ be a bounded domain of the form $D=\{z \mid V(z)<0\}, V(z)$ plurisubharmonic and continuous in a neighborhood of $D$. (This is a "smoothness" condition.) Then the Dirichlet problem is possible for the upper envelope $\phi(z)$ of the plurisubharmonic functions in $\bar{D}$ that are smaller or equal the given continuous boundary values $b(z)$ (at those points of $\partial D$ where $b(z)$ is defined) if and only if the boundary values $b(z)$ are prescribed on and only on the Silov boundary $S(D)$ of $D$.

For complex dimension one the conditions on $D$ imply that $S(D)=\partial D$ and hence we obtain as corollary that the (ordinary) Dirichlet problem is possible for domains $D=\{z \mid V(z)<0\}, V(z)$ subharmonic and continuous in a neighborhood of $D$.

For dimension higher than one we are faced with the problem to characterize the Šilov boundary. This has been done so far only for a rather special class of domains, the Reinhardt circular domains (de Leuuw [18], compare also Behnke-Stein [2]). We show: If $D=\{z \mid V(z)<0\}$ as above and $V(z)$ is $\left(C^{2}\right)$, then all those points on $\partial D$ where $\bar{\partial} \partial V=\sum\left(\partial^{2} V / \partial z_{\mu} \partial \bar{z}_{\nu}\right) d z_{\mu} d \bar{z}_{\nu}$ is positive definite belong to $S(D)$, and all points on $\partial D$ where in a neighborhood $\bar{\partial} \partial V=0$ for all directions do not belong to $S(D)$. In particular: If $D$ is strictly pseudoconvex then $S(D)=\partial D$ and if $D$ is an analytic polyhedron then $S(D)=$ "distinguished boundary surface of $D$ ".

The Silov boundary of any domain is equal to the Silov boundary of its envelope of holomorphy $E(D): S(D)=S(E(D))$.

Besides the plurisubharmonic solution $\phi(z)$ one can introduce analogously the plurisuperharmonic solution $\Phi(z)$ which exists if and only if $\phi(z)$ exists. Let $h(z)$ be the harmonic solution, then $\phi(z) \leqq h(z) \leqq \Phi(z)$ with equality in all points of $D$ if equality holds in one point. While $h(z)$ is not pseudo-conformally invariant, $\phi(z)$ and $\Phi(z)$ are, and provide an estimate: Let $D^{*}$ be pseudoconformally equivalent to $D=\{z \mid V(z)<0\}$, let $h(z)$ be harmonic in $D, h^{*}\left(z^{*}\right)$ in $D^{*}$ and $h(z)=h^{*}\left(z^{*}(z)\right)$ on $\partial D$, then $\left|h(z)-h^{*}\left(z^{*}(z)\right)\right| \leqq \Phi(z)-\phi(z)$.

The plurisubharmonic solutions have the property to be only subadditive, this excludes the representation of $\phi(z)$ in the form of an integral over the boundary values. Therefore the following property is important because it provides us with a different method to calculate or estimate $\phi(z):$ Let $b^{*}(z)$ 
$=b(z)$ on $S(D), b^{*}(z)=\max _{z \in S(D)} b(z)$ on $\bar{D}-S(D)$. Then the envelope of holomorphy $E(H)$ of the pointset

$$
H=\left\{(z, w)|z \in \bar{D},| w \mid<e^{-b^{*}(z)}\right\}
$$

is

$$
E(H)=\left\{(z, w)|z \in \bar{D},| w \mid<e^{-\phi(z)}\right\} .
$$

Both $H$ and $E(H)$ are schlicht. Therefore $E(H)$ can be calculated explicitly by a method described in Bremermann [13]. Hence $\phi(z)$ can be calculated.

It is shown that most results can be extended to Stein manifolds and some results to complex Banach spaces of infinite dimension. Also, the condition that the boundary values be continuous can be relaxed such that upper semicontinuous boundary values and the values of $\log |f|, f$ holomorphic, are admissible. The latter indicates the possibility of Nevanlinna-type applications. Such and other applications have been made for his extended class by S. Bergman $[5 ; 6]$ and it can be expected that some of these can be done for the plurisubharmonic solutions also. Furthermore it is expected that the results of this paper will permit to generalize parts of the Morse theory (Marston Morse [24]) to several variables.

Finally examples are given in the form of tube domains which are pseudoconvex if and only if they are convex. Here the Silov boundary is given exactly by the strictly convex points of the base.

The general idea of the plurisubharmonic solution (for the case of analytic polyhedra) has been outlined already in Bremermann [10].

I am indebted to Dr. L. Ehrenpreis for valuable advice.

2. Connection with the Bergman extended classes. S. Bergman has been the first to suggest to extend the class of pluriharmonic functions such that

(1) The (generalized) Dirichlet problem becomes uniquely solvable.

(2) The "extended class" contains the pluriharmonic functions (hence if the prescribed values belong to a pluriharmonic function, then this pluriharmonic function is the solution within the class).

(3) As many properties of the pluriharmonic functions as possible are preserved under the extension.

For this purpose Bergman has not considered general domains-for these no extended class has been introduced so far-but "domains with a distinguished boundary surface." These are domains bounded by a finite number of segments of analytic hypersurfaces. The union of the intersections of each $n$ of the hypersurfaces is the "distinguished boundary surface" (d.b.s.). A holomorphic function as well as a pluriharmonic function is determined already by its values on the d.b.s.

A function of an "extended class" is first defined on the whole boundary in the following way (only complex dimension 2 has been treated explicitly. 
The case of higher dimension is more difficult): The boundary of a domain with a d.b.s. in the space $C^{2}$ consists of families of nonintersecting analytic surfaces of complex dimension 1 with their boundaries on the d.b.s. For these the (complex) one-dimensional Dirichlet problem can be solved in the usual way and hence the function be defined on the whole boundary. The domain is then decomposed in a two parameter family of nonintersecting segments of analytic surfaces (of complex dimension one). The boundaries lie on the boundary of the domain and then for each analytic surface the one dimensional boundary value problem is solved and thus a function defined in the whole domain (compare a more detailed description for the case of analytic polyhedra in Bergman [5, pp. 150-152] and Bremermann [10]).

The process of the decomposition of the domain in a family of analytic surfaces is not unique and hence there are different extended classes.

In the case of an analytic polyhedron with $k$ defining functions there are in general $k$ different extended classes.

We show in 7.7 that the functions of Bergman's extended classes are larger or equal our plurisubharmonic solution. For a special case Bergman has already proved the existence of $\phi(z)$ and $\Phi(z)$ (Bergman [4]) and used them as estimates for his functions. At that time the theory of plurisubharmonic functions was in its infancy and very little known.

Besides this type of extended class Bergman has also defined a second type of extended class. Here the function is first defined on the whole boundary as above but then the harmonic solution of these boundary values is taken, and it applies what has been said in the introduction about the harmonic functions. Compare Bergman [4;5] and [6]. For a more complete bibliography on Bergman's work on his extended classes see Bergman [6]. Recently a third type of extended class for domains that permit a group of automorphisms has been considered by Lowdenslager [22, 22a].

3. Plurisubharmonic functions, pseudo-convex domains. 3.1. Plurisubharmonic functions and pseudo-convex domains have been studied extensively in recent years. (Compare Lelong [19], Bremermann [10].) We recall here mainly the definitions.

A real valued function $V(z)$ is plurisubharmonic in a domain $D$ if and only if the following conditions are satisfied:

(a) $-\infty \leqq V(z)<\infty$.

(b) $V(z)$ is upper semi-continuous.

(c) The restriction of $V(z)$ to any analytic plane $E=\left\{z \mid z=z_{0}+\lambda a\right\}$ (of complex dimension one) is subharmonic in the intersection $E \cap D$.

If $V(z)$ is twice continuously differentiable, then $V(z)$ is plurisubharmonic if and only if

$$
\bar{\partial} \partial V=\sum_{\mu, \nu=1}^{n} \frac{\partial^{2} V}{\partial z_{\mu} \partial \bar{z}_{\nu}} d z_{\mu} d \tilde{z}_{\nu} \geqq 0 .
$$


3.2. Pseudo-convex domains (Compare Bremermann [10]). A domain $D$ is pseudo-convex if and only if $D$ is of the form

$$
D=\lim D_{\nu}, \quad D_{\nu} \subset D_{\nu+1} \subset D,
$$

where

$$
D_{\nu}=\left\{z \mid V_{\nu}(z)<0\right\},
$$

where $V_{\nu}(z)$ is plurisubharmonic in a neighborhood of $D_{\nu}$.

3.3. A domain $D$ is strictly pseudo-convex if and only if $D$ is of the form

$$
D=\{z \mid V(z)<0\},
$$

where $V(z)$ is plurisubharmonic in a neighborhood of $D$ and

$$
\bar{\partial} \partial V=\sum_{\mu, \nu=1}^{n} \frac{\partial^{2} V}{\partial z_{\mu} \partial \bar{z}_{\nu}} d z_{\mu} d \bar{z}_{\nu}
$$

is positive definite on the boundary of $D$.

4. The generalized Dirichlet problem for strictly pseudo-convex domains.

4.1. Theorem. Let $D$ be a bounded strictly pseudo-convex domain. Let $b(z)$ be continuous real values prescribed on the boundary $\partial D$ of $D$. Then the upper envelope $\phi(z)$ of all functions plurisubharmonic in $\bar{D}$ that are smaller or equal $b(z)$ on $\partial D$ exists and is plurisubharmonic in $D$ and assumes the given boundary values, that means for any sequence $z_{\nu} \rightarrow z_{0}, z_{\nu} \in D, z_{0} \in \partial D$ we have $\lim \sup \phi\left(z_{\nu}\right)$ $=b\left(z_{0}\right)$.

Proof. Let us denote the family of functions that are plurisubharmonic in $\bar{D}$ and smaller or equal $b(z)$ on $\partial D$ by $\mathfrak{R}(b(z), D)$.

(1) $\mathfrak{R}(b(z), D)$ is bounded from above in $D$. Therefore $\phi(z)$ exists and is plurisubharnonic as upper envelope of an upper bounded family of plurisubharmonic functions.

(2) $\phi(z) \geqq b(z)$ on $\partial D$.

$D$ can by assumption be represented in the form

$$
D=\{z \mid V(z)<0\},
$$

$V(z)$ plurisubharmonic in a neighborhood of $D$.

We now consider the function

$$
V^{*}(z)=V(z)-\epsilon\left\|z-z_{0}\right\|^{2}
$$

where $\|z\|^{2}=\left|z_{1}\right|^{2}+\cdots+\left|z_{n}\right|^{2}, \epsilon>0$ and constant. We have

$$
\bar{\partial} \partial \epsilon \mid z-z_{0} \|^{2}=\epsilon \sum_{\mu=1}^{n} d z_{\mu} d \bar{z}_{\mu} .
$$

$D$ is by assumption strictly pseudo-convex, that means $\bar{\partial} \partial>0$ in $\bar{I}$, and is bounded. Hence there exists a $\delta>0$ such that 


$$
\bar{\partial} \partial V>\delta \sum_{\mu=1}^{n} d z_{\mu} d \bar{z}_{\mu} \text { in } \bar{D} .
$$

Thus if we make $\epsilon<\delta$, then $V^{*}(z)$ is still a plurisubharmonic function in $\bar{D}$.

We have $V^{*}\left(z_{0}\right)=0$ and

$$
V^{*}(z)<0 \text { in } \bar{D}-\left\{z_{0}\right\} .
$$

If we multiply $V^{*}(z)$ by a constant $c>0$, then $c V^{*}(z)$ has the same properties as $V^{*}(z)$, and by taking $c$ large enough we achieve that

$$
c V^{*}(z)+b\left(z_{0}\right)<b(z)+\epsilon^{\prime} \text { on } \partial D
$$

for arbitrary small $\epsilon^{\prime}>0$.

Let $V\left(z_{0}, \epsilon^{\prime} ; z\right)=c V^{*}(z)+b\left(z_{0}\right)-\epsilon^{\prime}$, then this function has the property

$$
V\left(z_{0}, \epsilon^{\prime} ; z\right)<b(z) \text { on } \partial D
$$

and

$$
V\left(z_{0}, \epsilon^{\prime} ; z_{0}\right)=b\left(z_{0}\right)-\epsilon^{\prime} .
$$

Hence the upper envelope $\psi_{\epsilon^{\prime}}(z)$ of the family

$$
\left\{V\left(z_{0}, \epsilon^{\prime} ; z\right) \text { for all } z_{0} \in \partial D\right\}
$$

satisfies

$$
b(z)-\epsilon^{\prime} \leqq \psi_{\epsilon^{\prime}}(z) \leqq b(z) \text { for } z \in \partial D
$$

and every $\epsilon^{\prime}>0$.

The class $V\left(z_{0}, \epsilon^{\prime} ; z\right), z_{0} \in \partial D, \epsilon^{\prime}>0$ is a subclass of $\mathfrak{R}(b(z), D)$.

Consequently

$$
\phi(z)=\text { upper envelope of } \mathfrak{l}(b(z), D)
$$

has the property

$$
\phi(z) \geqq b(z) \quad \text { for } z \in \partial D .
$$

(3) It is not trivial to conclude that

$$
\phi(z) \leqq b(z) \text { on } \partial D .
$$

EXAmple. Let us take the punctured unit disc $\{z|0<| z \mid<1\}$ in the plane of one complex variable. Let $b(z) \equiv 1$ on $|z|=1$ and $b(0)=0$. Then there exist plurisubharmonic $=$ subharmonic functions (for dimension one) that assume these boundary values (for example $|z|$ ), however the upper envelope of all subharmonic functions smaller than or equal to $b(z)$ on the boundary is the function $\phi(z) \equiv 1$, which violates $\phi(z) \leqq b(z)$.

Let $V^{* *}(z)=-V^{*}(z)$, then $V^{* *}(z)$ is plurisuperharmonic. The plurisuperharmonic functions are a subclass of the superharmonic functions. Hence $V^{* *}$ has the properties 
(1) $V^{* *}(z)$ is superharmonic in $\bar{D}$,

(2) $V^{* *}\left(z_{0}\right)=0$,

(3) $V^{* *}(z)>0$ on $\bar{D}-\left\{z_{0}\right\}$.

A function with such properties is a barrier (comp. Kellog [17]). Thus a barrier exists for every boundary point $z_{0}$ of $D$. As a consequence the Dirichlet problem for harmonic functions is solvable for $D$ (and continuous boundary values (comp. Kellog [17]). Hence there exists a harmonic function $h(z)$ in $D$, continuous in $\bar{D}$ assuming the given boundary values $b(z)$.

Now every $\psi(z) \in \mathfrak{R}(b(z), D)$ is plurisubharmonic and therefore in particular subharmonic in $\bar{D}$.

We have $\psi(z) \leqq b(z)=h(z)$ on $\partial D$, and hence, by the fundamental property of the subharmonic functions the same inequality holds also in $D$.

Hence $\psi(z) \leqq h(z)$ in $\bar{D}$ and therefore

$$
\sup _{\psi \in \mathbb{R}}\{\psi(z)\} \leqq h(z) \text { in } \bar{D} \text {. }
$$

Now $h(z)$ is continuous in $\bar{D}$, hence

$$
\phi(z)=\lim _{z^{\prime} \rightarrow z} \sup _{\psi \in \mathbb{R}}\left\{\psi\left(z^{\prime}\right)\right\} \leqq h(z) \text { in } \bar{D}
$$

and therefore

$$
\phi(z) \leqq b(z) \text { on } \partial D .
$$

Under (2) we have proved that

$$
\phi(z) \geqq b(z) \text { on } \partial D,
$$

therefore

$$
\phi(z)=b(z) \text { on } \partial D,
$$

and in particular $\lim \sup _{z^{\prime} \rightarrow z} \phi\left(z^{\prime}\right)=b(z)$ on $\partial D$ for any sequence $z^{\prime} \rightarrow z, z \in \bar{D}$, $z^{\prime} \in D$. q.e.d.

5. The generalized Dirichlet problem for analytic polyhedra. An analytic polyhedron $\mathfrak{B}$ is given by $k$ functions $f_{1}(z) \cdots f_{k}(z), k \geqq n$, that are holomorphic in a domain $D$ such that

$$
\mathfrak{B}=\left\{z|| f_{1}(z)|<1, \cdots,| f_{k}(z) \mid<1, z \in D\right\},
$$

and $\mathfrak{B C} \subset D$.

Instead of $\left|f_{j}(z)\right|<1$ one could consider $f_{j}(z) \in \Delta_{j}$ where $\Delta_{j}$ is a domain in the complex plane. We will limit ourselves to the above type. Any domain of holomorphy can be approximated by such analytic polyhedra.

Let

$$
\sigma_{j_{1} \cdots j_{n}}=\partial \mathfrak{B} \cap\left\{z|| f_{j_{1}}(z)|=1, \cdots,| f_{j_{n}}(z) \mid=1\right\} .
$$

Then the "distinguished boundary surface" of $\mathfrak{B}$, in the following denoted

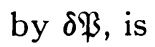




$$
\delta \mathfrak{P}=\underset{\left(j_{1} \cdots j_{n}\right) \in A}{U} \sigma_{j_{1} \cdots j_{n}},
$$

where $A$ is the set of all $n$-tuples with $j_{1}<j_{2}<\cdots<j_{n}$ formed from $n$ different numbers $j_{i} \in\{1, \cdots, k\}$.

5.1. Among the pseudo-convex domains the analytic polyhedra are in a way the opposite of the strictly pseudo-convex domains. Let $V(z)$ $=\sup \left\{\log \left|f_{1}(z)\right|, \cdots, \log \left|f_{k}(z)\right|\right\}$, then $\mathfrak{B}=\{z \mid V(z)<0, z \in D\}$, and $\bar{\partial} \partial V$ is zero everywhere on the boundary with the exception of the distinguished boundary surface.

5.2. Let none of the functions $f_{j}$ defining $\mathfrak{P}$ be a constant. Then any function that is plurisubharmonic in $\mathfrak{B}$ and smaller than a constant $M$ on $\delta \mathfrak{B}$ is smaller than $M$ in $\mathfrak{B}$. In other words the maximum principle holds for $\delta \mathfrak{B}$ and $\mathfrak{B}$. Note that the moduli of holomorphic functions are in particular plurisubharmonic, and hence the maximum principle holds for them.

Let

$$
\begin{aligned}
\sigma_{j} & =\overline{\mathfrak{P}} \cap\left\{z|| f_{j}(z) \mid=1\right\}, \\
\sigma_{j_{1} j_{2}} & =\overline{\mathfrak{P}} \cap\left\{z|| f_{j_{1}}(z)|=1,| f_{j_{2}}(z) \mid=1\right\}
\end{aligned}
$$

etc. till $\sigma_{j_{1}} \ldots j_{n}$. As none of the $f_{j}$ is a constant, $\left\{z|| f_{j}(z) \mid=1\right\}$ is of real dimension $2 n-1$. Now if $\sigma_{j}$ is of dimension $2 n-1$ then it is composed of a collection of analytic surfaces

$$
\overline{\mathfrak{B}} \cap\left\{z \mid f_{j}(z)=e^{i v}\right\} .
$$

$f_{j}(z)$ is holomorphic in a neighborhood $D$ of $\mathfrak{P}$. Then the analytic variety $f_{j}(z)=e^{i \vartheta}$ has no boundary points in the interior of $D$. Therefore the boundary of

$$
\overline{\mathfrak{B}} \cap\left\{z \mid f_{j}(z)=e^{i \vartheta}\right\} \text { is on } \underset{\rho=1, \cdots, k ; \rho \neq j}{\bigcup} \sigma_{j, \rho} .
$$

If $\sigma_{j}$ is not of dimension $2 n-1$, then it must be empty or it must be a subset of $U_{\rho=1}, \ldots, k ; \rho \neq j=1, \sigma_{j, \rho}$.

A plurisubharmonic function that is smaller or equal $M$ on $\bigcup_{\rho=1, \cdots, k ; \rho \neq j} \sigma_{j, \rho}$ is smaller or equal $M$ on $\sigma_{j}$ because the maximum principle (compare Bremermann $[10$, p. 22]) holds for analytic surfaces and plurisubharmonic functions (even if $\sigma_{j}$ has singularities because the singularities are analytic surfaces of lower dimension or isolated points).

We can repeat the conclusion for $\sigma_{j_{1} j_{2}}$, etc. till we reach $\sigma_{j_{1}} \cdots j_{n}$. Here we have to stop because in general the intersection of the zeros of $n$ holomorphic functions is no longer an analytic surface without boundary in $D$ but consists of isolated points. Hence we conclude.

If $V \leqq M$ on $\delta \mathfrak{B}$, then $V \leqq M$ on $\delta \mathfrak{P}$ and hence $V \leqq M$ in $\overline{\mathfrak{P}}$.

5.3. THEOREM. Let $\mathfrak{P}$ be an analytic polyhedron such that all the sets $\left\{z \mid f_{j_{1}}(z)=e^{i \vartheta_{i_{1}}}, \cdots, f_{j_{n}}(z)=e^{i \vartheta_{j_{n}}, z} \in D\right\}$ are of dimension zero or empty for any 
$n$-tuple $j_{1} \cdots j_{n}$ with $j_{1}<\cdots<j_{n}$ and $0 \leqq \vartheta_{j_{1}} \leqq 2 \pi, \cdots, 0 \leqq \vartheta_{j_{n}} \leqq 2 \pi$. Let $b(z)$ be continuous boundary values prescribed on $\delta \mathfrak{~}$. Then the upper envelope $\phi(z)$ of the class of all functions that are plurisubharmonic in $\mathfrak{P}$ and smaller or equal $b(z)$ on $\delta \mathfrak{B}$ exists and assumes the values $b(z)$ in the sense that $\lim \sup \phi\left(z^{v}\right)=b\left(z_{0}\right)$ for every sequence $z^{\nu} \rightarrow z_{0}, z^{\nu} \in \mathfrak{B}, z_{0} \in \delta \mathfrak{B}$.

(1) The existence of $\phi(z)$ follows from the fact that the maximum principle holds for $\delta \mathfrak{P}$ and $\overline{\mathfrak{P}}$ as proved in 5.2.

(2) $\phi(z) \geqq b(z)$ for $z \in \delta \Re$ :

Let $z_{0} \in \delta \mathfrak{B}$, then $f_{j_{1}}\left(z_{0}\right)=e^{i \vartheta_{j_{1}}}, \cdots, f_{j_{n}}\left(z_{0}\right)=e^{i \vartheta j_{n}}$. Let $\chi(\xi)=(\xi+a / 1+a)$, $a$ real and $a>0$.

Let us denote $\chi\left(f_{j}(z) / e^{i \vartheta_{i}}\right)$ by $g_{j}(z)$, then

$$
g_{j}(z)=1 \text { for } z \in\left\{z^{\prime} \mid f_{j}\left(z^{\prime}\right)=e^{i \vartheta_{j}}\right\}
$$

and

$$
\left|g_{j}(z)\right|<1 \text { on } \overline{\mathfrak{P}}-\left\{z \mid f_{j}(z)=e^{i \vartheta_{j}}\right\}
$$

hence if $U(z)=\log \left|g_{j_{1}}(z)\right|+\cdots+\log \left|g_{\rho_{n}}(z)\right|$ we have

$U(z)$ is plurisubharmonic in $\overline{\mathfrak{P}}$

and

$$
U(z)=0 \text { for } z \in S=\left\{z \mid f_{j_{1}}(z)=e^{i \vartheta_{i_{1}}}, \cdots, f_{j_{n}}(z)=e^{i \vartheta_{j_{n}}}\right\}
$$

and

$$
U(z)<0 \text { for } z \in \overline{\mathfrak{B}}-S .
$$

Because of our assumption that $S \cap D$ is zero dimensional (or empty) there are besides $z_{0}$ at most finitely many points $z^{(1)}, \cdots, z^{(s)}$ of $S$ on $\delta \mathfrak{P}$.

Through each one of the points $z^{(1)}, \cdots, z^{(s)}$ we take an analytic plane of dimension $n-1$ that does not contain $z_{0}$. These planes can be written in the form

$$
\begin{gathered}
\left\{z \mid l_{\nu}(z)=0\right\}, \text { where } \\
l_{\nu}(z)=a_{\nu_{1}} z_{1}+\cdots+a_{\nu_{n}} z_{n}+a_{\nu_{0}}
\end{gathered}
$$

$\left(z_{1} \cdots z_{n}\right.$ the coordinates of $\left.z\right)$. Then the sum

$$
\log \left|l_{1}(z)\right|+\cdots+\log \left|l_{s}(z)\right|
$$

is $-\infty$ at the points $z^{(1)}, \cdots, z^{(s)}$ but finite at $z_{0}$. We add a constant $c_{0}$ such that the sum has the value zero at $z_{0}$.

By multiplying the functions $\log \left|g_{j_{1}}(z)\right|, \cdots, \log \left|g_{j_{n}}(z)\right|$ with positive real constants $c_{j_{1}}, \cdots, c_{j_{n}}$ sufficiently large we achieve that the function

$$
\begin{aligned}
U^{*}(z)= & c_{j_{1}} \log \left|g_{j_{1}}(z)\right|+\cdots+c_{j_{n}} \log \left|g_{j_{n}}(z)\right|+\log \left|l_{1}(z)\right|+\cdots \\
& +\log \left|l_{s}(z)\right|+c_{0}
\end{aligned}
$$


has the property that

(1) $U^{*}(z)$ is plurisubharmonic in $\overline{\mathfrak{B}}$,

(2) $U^{*}\left(z_{0}\right)=0$,

(3) $U^{*}(z)<0$ on $\overline{\mathfrak{P}}-\left\{z_{0}\right\}$.

From here $\phi(z) \geqq b(z)$ on $\delta \mathfrak{P}$ follows exactly as in 4.1 .

We note for an application in $\$ 6$ that we can choose the constants $c_{j_{1}}, \cdots, c_{j_{n}}$ as integers. Therefore $e^{U^{*}(z)}$ is the modulus of a holomorphic function:

$$
e^{U^{*}(z)}=|h(z)|=\left|\prod_{\nu=1}^{n}\left(g_{j_{\nu}}(z)\right)^{c_{j}} \cdot \prod_{\mu=1}^{s} l_{\mu}(z) \cdot e^{c_{0}}\right| .
$$

We have

(a) $h(z)$ is holomorphic in $\overrightarrow{\mathfrak{B}}$,

(b) $\left|h\left(z_{0}\right)\right|=1$,

(c) $|h(z)|<1$ on $\overline{\mathfrak{P}}-\left\{z_{0}\right\}$.

(3) $\phi(z) \leqq b(z)$.

For every $\epsilon>0$ we can find a constant $c>0$ such that the function

$$
U^{* *}(z)=-c U^{*}(z)+b\left(z_{0}\right)+\epsilon
$$

has the property

$$
U^{* *}(z) \geqq b(z) \text { on } \delta \mathfrak{B} .
$$

Let $\psi(z)$ be any function in the class of functions that are plurisubharmonic in $\overline{\mathfrak{P}}$ and smaller or equal $b(z)$ on $\delta \mathfrak{B}$, then

$$
\psi(z)-U^{* *}(z) \leqq 0 \text { on } \delta \mathfrak{B} .
$$

Now $\psi(z)-U^{* *}(z)$ is plurisubharmonic, hence because the maximum principle holds for $\delta \mathfrak{P}$ and $\overline{\mathfrak{B}}$ we have that the inequality holds also in $\overline{\mathfrak{B}}$, hence

$$
\psi(z) \leqq U^{* *}(z) \text { in } \overline{\mathfrak{P}} \text {. }
$$

Then the same inequality holds also for $\phi(z)$, the upper envelope of all $\psi(z) . U^{* *}(z)$ is continuous, therefore

$$
\lim _{z^{\nu} \rightarrow z_{0}} \sup \phi\left(z^{\nu}\right) \leqq U^{* *}\left(z_{0}\right)=b\left(z_{0}\right)+\epsilon .
$$

This holds for every $\epsilon>0$, hence

$$
\lim _{z^{\nu} \rightarrow z_{0}} \sup \phi\left(z^{\nu}\right) \leqq b\left(z_{0}\right)
$$

for arbitrary $z_{0} \in \delta \mathfrak{P}$ and $z^{\nu} \in \mathfrak{P}$. q.e.d.

6. The Silov boundary. Summarizing the results of $\S 4$ and 5: We have seen that the Dirichlet problem can be solved by plurisubharmonic functions:

(a) for strictly pseudo-convex domains and the values prescribed on the whole boundary 
(b) for analytic polyhedra and the values prescribed on the distinguished boundary surface only.

We will see in $\$ 7$ that in the case of more general domains we have to prescribe the boundary values on no more and no less than a certain subset of the boundary which in the theory of Banach algebras (Mirkil [23], ArensSinger [1]) is known as the "Šilov boundary." Thus we are led to determine the Silov boundary of a domain, a problem which is also of interest for Banach algebras (de Leuuw [18]). A rather general though not exhaustive solution is given in the following.

6.1. The Silov boundary $S$ of a domain $D$ is the smallest closed subset of $\partial D$ such that for every function $f$ holomorphic in $D$ and continuous in $\bar{D}$ we have

$$
\text { if }|f| \leqq M \text { on } S \text {, then }|f| \leqq M \text { in } \bar{D} .
$$

In other words $S$ is the smallest closed subset of $\bar{D}$ such that the maximum principle holds for $S, \bar{D}$ and the class of moduli of holomorphic functions in $\bar{D}$. For a proof of the existence and uniqueness of the Silov boundary see Loomis $[21$, p. 80]. (A similar though not identical notion has also been introduced by Bergman [7], who called "Maximumfläche" any subset of the boundary $\partial D$ of a domain $D$ such that the maximum principle holds for $\partial D$ and $\bar{D}$ with respect to the functions that are holomorphic in a neighborhood of $D$ ).

6.2. To prove 6.3 we need the following lemma (which holds also for Stein manifolds) (Bremermann $[8$, p. 10 and p. $61 ; 14]$ ):

Lemma. Let $U(z)$ be plurisubharmonic and continuous in $G$. Then for any $\tilde{G} \subset \subset G$ and for any $\epsilon>0$ there exist $k$ functions $f_{1}, \cdots, f_{k}$ holomorphic in $G$ and $k$ positive constants $c_{1}, \cdots, c_{k}$ such that

$$
\text { for } z \in \tilde{G}: U(z)-\epsilon \leqq \sup \left\{c_{1} \log \left|f_{1}(z)\right|, \cdots, c_{k} \log \left|f_{k}(z)\right|\right\} \leqq U(z) \text {. }
$$

6.3. Theorem. Let $D$ be a bounded strictly pseudo-convex domain, then the Silov boundary is identical with the whole boundary of $D$.

Proof. Let $z_{0}$ be an arbitrary point on $\partial D$. Let $V^{*}(z)$ be the function of 4.1 , that means $V^{*}(z)$ is plurisubharmonic in $\bar{D}, V^{*}\left(z_{0}\right)=0$ and $V^{*}(z)<0$ for $z \in \bar{D}-\left\{z_{0}\right\}$.

If we multiply $V^{*}(z)$ by a constant $c>0$ we can make $c V^{*}(z)$ smaller $-2 \epsilon$ outside of the intersection of an arbitrarily small neighborhood $N\left(z_{0}\right)$ of $z_{0}$ with $\bar{D}$.

Now we apply our lemma with $U(z)=c V^{*}(z)$ and $\widetilde{G}$ a neighborhood of $\bar{D}$. Then one of the functions $f_{1} \cdots f_{k}$, let it be $f_{j_{0}}$, has the property that

$$
c V^{*}\left(z_{0}\right)-\epsilon \leqq c_{j_{0}} \log \left|f_{j_{0}}\left(z_{0}\right)\right| \leqq c V^{*}\left(z_{0}\right),
$$

and for $z \in \bar{D}: c_{j_{0}} \log \left|f_{j_{0}}(z)\right| \leqq c V^{*}(z)$. Hence 


$$
-\epsilon \leqq c_{j_{0}} \log \left|f_{j_{0}}\left(z_{0}\right)\right| \leqq 0
$$

and

$$
c_{j_{0}} \log \left|f_{j_{0}}(z)\right|<-2 \epsilon \text { for } z \in \bar{D}-N\left(z_{0}\right) .
$$

Thus $c_{j_{0}} \log \left|f_{j_{0}}(z)\right|$ assumes its maximum with respect to $\bar{D}$ in $N\left(z_{0}\right)$. Hence $N\left(z_{0}\right)$ contains points that belong to the Silov boundary of $D$. This is true for arbitrarily small neighborhoods $N\left(z_{0}\right)$ of $z_{0}$, therefore and because the Silov boundary is a closed set, $z_{0}$ belongs to it. $z_{0}$ was an arbitrary point on $\partial D$. Hence $\partial D=$ Silov boundary of $D$.

6.4. Let $\mathfrak{B}$ be an analytic polyhedron satisfying the conditions of 5.3. Then the Silov boundary is identical with the distinguished boundary $\delta \mathfrak{B}$ of $\mathfrak{B}$.

The function $h(z)$ constructed in the proof of 5.3 has the properties (for an arbitrary point $\left.z_{0} \in \delta \mathfrak{B}\right)$.

(1) $h(z)$ is holomorphic in $\mathfrak{B}$.

(2) $\left|h\left(z_{0}\right)\right|=1$.

(3) $|h(z)|<1$ on $\overline{\mathfrak{B}}-\left\{z_{0}\right\}$.

Hence $z_{0}$ belongs to the Silov boundary of $\mathfrak{B}$, which we denote in the following by $S(\mathfrak{B})$. Therefore

$$
\delta \mathfrak{S} \subseteq S(\mathfrak{P}) .
$$

On the other hand it has been shown in $\mathbf{5 . 2}$ that the maximum principle holds for $\delta \mathfrak{P}$ and $\overline{\mathfrak{P}}$ and the class of moduli of functions holomorphic in $\overline{\mathfrak{P}}$. But it is easy to see that the max principle holds also for $\delta \mathfrak{P}$ and $\overline{\mathfrak{P}}$, and the class of moduli of functions that are holomorphic in $\mathfrak{P}$ and continuous in $\overline{\mathfrak{P}}$, because according to the Oka-Weil theory the functions holomorphic in $\mathfrak{P}$ can be approximated by functions holomorphic in a neighborhood of $\mathfrak{B}$. Hence

$$
S(\mathfrak{P}) \subseteq \delta \mathfrak{B}
$$

and therefore

$$
S(\mathfrak{P})=\delta \mathfrak{P}
$$

6.5. Thus we have determined the Silov boundary for two important classes of domains of holomorphy.

The problem to determine the Silov boundary of an arbitrary domain cannot be solved simply by an approximation of the given domain by domains of a simple type whose Šilov boundary is known. Any domain of holomorphy can be approximated by strictly pseudo-convex domains as well as by analytic polyhedra. (In particular we can approximate an analytic polyhedron by strictly pseudo-convex domain.) This shows that it is possible that for a sequence $D_{\nu} \rightarrow D$ we have $\lim S\left(D_{\nu}\right) \neq S(D)$.

Thus the problem to determine the Silov boundary of domains other than strictly pseudo-convex domain and analytic polyhedra requires further investigations. Before these we should like to note the following: 
Lemma. Let $\left\{D_{\nu}\right\}$ be a sequence with $D_{\nu} \subset D_{\nu+1} \subset D, \lim D_{\nu}=D, D$ bounded. Then we have for the Šlov boundaries

$$
\lim S\left(D_{v}\right) \supseteq S(D) .
$$

Proof. Let $f$ be any function holomorphic in $D$, continuous in $\bar{D}$.

Let $\nu$ be so large that if $|f| \leqq M$ on $\lim S\left(D_{\nu}\right)$ then $|f| \leqq M+\epsilon / 2$ on $S\left(D_{\nu}\right)$ and hence on $\partial D_{\nu}$, and if $|f| \leqq M+\epsilon / 2$ on $\partial D_{\nu}$, then $|f| \leqq M+\epsilon$ on $\partial D$.

This obviously is possible because $D$ is bounded and $f$ is continuous in $\bar{D}$. Hence we have: If $|f| \leqq M$ on $\lim S\left(D_{\nu}\right)$, then $|f| \leqq M+\epsilon$ on $\partial D$. This holds for every $\epsilon>0$, hence the maximum principle holds for $\lim S\left(D_{\nu}\right)$ and $\partial D$. This implies

$$
\lim S\left(D_{v}\right) \supseteq S(D) .
$$

As we have seen $S(D)$ can actually be smaller than $\lim S\left(D_{\nu}\right)$.

6.6. In treating the problem to determine the Silov boundary of arbitrary domains $D$ two cases have to be distinguished.

(1) $D$ is not a domain of holomorphy.

(2) $D$ is a domain of holomorphy.

The following lemma reduces Case 1 to Case 2 and to the problem of determining the envelope of holomorphy of $D$. (The envelope of holomorphy can be calculated by a process described in Bremermann [13].)

Theorem. The Šilov boundary $S(D)$ of a bounded domain $D$ is equal to the Silov boundary of the envelope of holomorphy $E(D)$ of $D$ :

$$
S(D)=S(E(D))
$$

Corollary.

$$
S(E(D)) \subseteq \partial D \cap \partial E(D)
$$

Proof. Let

$$
\begin{aligned}
& \Delta_{1}=\partial E(D) \cap \partial D, \\
& \Delta_{2}=\partial D-\Delta_{1} .
\end{aligned}
$$

Let $f(z)$ be an arbitrary function holomorphic in $D$ and continuous in $\bar{D}$. Let $\max _{z \in D}|f|=M$. Suppose $|f|$ would assume its maximum with respect to $\bar{D}$ at the point $z_{0} \in \Delta_{2}$. Then we would have

$$
f\left(z_{0}\right)=M e^{i \vartheta} .
$$

Now $f(z)$ is holomorphic on $\Delta_{2}$ as $\Delta_{2} \subset E(D)$ hence $f(z)=M e^{i \vartheta}$ is an analytic surface in $E(D)$, that does not intersect $D$ (unless $f=$ const.)

Then the function

$$
\left(f(z)-M e^{i \vartheta}\right)^{-1}
$$


would be holomorphic in $D$ but have a pole at the point $z_{0}$ in $E(D)$. This is not possible because any function holomorphic in $D$ is holomorphic in $E(D)$.

Therefore $f(z)$ assumes its maximum in $\bar{D}$ on $\Delta_{1}$, unless $f(z)=$ constant. Hence

$$
\text { if }|f(z)| \leqq M \text { on } \Delta_{1}, \text { then }|f(z)| \leqq M \text { in } \bar{D},
$$

and it follows

$$
S(D) \subseteq \Delta_{1} .
$$

Any function $g(z)$ holomorphic in $D$ assumes in $E(D)$ only values that it assumes already in $D$ (otherwise $(g(z)-a)^{-1}$ would be holomorphic in $D$ but not in $E(D))$. Therefore if $g(z)$ is holomorphic in $E(D)$ and continuous in $\bar{E}(D)$, and

$$
\text { if }|g(z)| \leqq M \text { on } S(D), \text { then }|g(z)| \leqq M \text { in } E(D),
$$

and because of continuity

$$
|g(z)| \leqq M \text { in } \bar{E}(D) .
$$

It follows

$$
S(E(D)) \subseteq \Delta_{1} \quad \text { and } \quad S(D) \supseteq S(E(D)) .
$$

On the other hand it is trivial that $S(D) \subseteq S(E(D))$, hence we have

$$
S(D)=S(E(D)) .
$$

6.7. After having reduced the Case 1 to the case of domains of holomorphy we have to determine the Šilov boundaries of the latter.

We consider in the following bounded domains of holomorphy $D$ that can be represented in the form

$$
D=\{z \mid V(z)<0\},
$$

where $V(z)$ is plurisubharmonic in a neighborhood of $D$. This class of domains of holomorphy (pseudo-convex domains) is rather general. (The most general domains of holomorphy $H$ (pseudo-convex domains) are limits of monotone increasing sequences of domains of the above type. (Compare Bremermann $[10])$.

6.8. Theorem. Let $D$ be bounded and let $D=\{z \mid V(z)<0\}$, where $V(z)$ is plurisubharmonic in a neighborhood of $D$. Then all those points of $\partial D$ where $\bar{\partial} \partial V$ exists in a neighborhood and is positive definite belong to the Šilov boundary $S(D)$.

Proof. Let $z_{0} \in \partial D$ and $\bar{\partial} \partial V$ at $z_{0}$ be positive definite. Then $\bar{\partial} \partial V$ is positive definite in a neighborhood of $z_{0}$. Then

$$
V(z)-\epsilon\left\|z-z_{0}\right\|^{2}
$$


is for $\epsilon$ sufficiently small still plurisubharmonic in a neighborhood of $z_{0}$ in particular for $\left\{z \mid\left\|z-z_{0}\right\| \leqq r\right\}$, for some $r$ sufficiently small.

Let now $u(z)$ be a $\left(C^{2}\right)$ function that coincides with $c\left\|z-z_{0}\right\|^{2}$ for $\left\|z-z_{0}\right\|$ $\leqq r / 2$, where $c$ is some positive constant, and such that

$$
u(z)=\text { const. }
$$

for $\left\|z-z_{0}\right\| \geqq r$, such that

$$
\bar{\partial} \partial u \leqq \bar{\partial} \partial\left\|z-z_{0}\right\|^{2}=\epsilon \sum_{\mu=1}^{n} d z_{\mu} d \bar{z}_{\mu} .
$$

Such a function $u(z)$ obviously exists. Then

$$
V^{*}(z)=V(z)-u(z)\left\|z-z_{0}\right\|^{2}
$$

has the following properties:

(1) $V^{*}(z)$ is plurisubharmonic and continuous in a neighborhood of $D$.

(2) $V^{*}\left(z_{0}\right)=0$.

(3) $V^{*}(z)<0$ in $\bar{D}-\left\{z_{0}\right\}$.

Thus the function $V^{*}(z)$ constructed here has the same properties as the function-denoted by the same symbol-used in 6.3, and hence, as has been proved there, there exists a function holomorphic in $\bar{D}$ that assumes its maximum with respect to $\bar{D}$ in an arbitrary small neighborhood $N\left(z_{0}\right)$ and is smaller in $\bar{D}-N\left(z_{0}\right) \cap \bar{D}$. It follows that $z_{0}$ belongs to $S(D)$.

6.9. Theorem. Let $D$ satisfy the conditions of 6.7 , let $V$ be of $\left(C^{2}\right)$, and let $z_{0}$ be such that $\bar{\partial} \partial V=0$ for all directions in a neighborhood of $z_{0}$. Then $z_{0} \notin S(D)$.

Under the assumptions made $V(z)$ is pluriharmonic in a neighborhood of $z_{0}$ and can be written $V(z)=\log |g|$, where $g(z) \neq 0$ and holomorphic in a neighborhood of $z_{0}$.

Then, if $r$ small enough, we have through every point of $\{z \mid V(z)=0\}$ $\cap\left\{z \mid\left\|z-z_{0}\right\|=r\right\}$ an analytic surface $g(z)=e^{i \vartheta}$ whose boundary is totally on the intersection of $\partial D$ and $\left\{z \mid\left\|z-z_{0}\right\|<r\right\}$. For these analytic surfaces the maximum principle holds. Hence any function holomorphic in $D$ and continuous in $\bar{D}$ that is smaller than a constant $M$ on

$$
\partial D \cap\left\{z \mid\left\|z-z_{0}\right\|=r\right\}
$$

is smaller $M$ in all points of

$$
\partial D \cap\left\{z \mid\left\|z-z_{0}\right\| \leqq r\right\} .
$$

Hence

$$
z_{0} \notin S(D) .
$$

REMARK. 6.8 and 6.9 give almost a complete characterization of the Silov boundary. As we have shown above 


$$
\partial D \cap\{z \mid \bar{\partial} \partial V>0\} \subset S(D) .
$$

Now $S(D)$ is a closed set. Therefore, if there exist points where $\bar{\partial} \partial V=0$ for some direction on the boundary of $D$, then $S(D)$ will contain such points. Examples can be given (tube domains) that show that the points where $\bar{\partial} \partial V=0$ can even fill an analytic surface.

However, we conjecture: Those points $z_{0} \in \partial D$ where $\bar{\partial} \partial V$ is zero for some complex direction, and where the same is true also for $z$ in a neighborhood of $z_{0}$, do not belong to $S(D)$.

In the following we note some properties of the Silov boundary that are useful for the next section.

6.10. Let $z_{0} \in S(D)$. Then there exists a function $f$ holomorphic in $D$ and continuous in $\bar{D}$ that assumes its maximum with respect to $\bar{D}$ in $\partial D \cap N\left(z_{0}\right)$, where $N\left(z_{0}\right)$ is an arbitrary small neighborhood of $z_{0}$, and $f$ is smaller than its maximum in $\bar{D}-N\left(z_{0}\right)$.

This follows immediately from the definition of the Silov boundary.

6.11. If $D$ is of the form $D=\{z \mid V(z)<0\}, V(z)$ plurisubharmonic in a neighborhood of $D$, then any function holomorphic in $D$ can be approximated in any $D_{0}$ that is relative compact in $D$ by functions holomorphic in a neighborhood of $D$. This follows from the fact that $V(z)$ is plurisubharmonic in a neighborhood, and hence the functions in $D$ can be approximated by functions in $\{z \mid V(z)<\epsilon\}$ for $\epsilon$ sufficiently small according to a result by Bremermann [14]. Then, however, we can also approximate the functions that are holomorphic in $D$ and continuous in $\bar{D}$ in all of $\bar{D}$. Hence we have

LeммA. If $D$ is of the form $D=\{z \mid V(z)<0\}$, then 6.10 is true already for a function holomorphic in a neighborhood of $D$.

6.12. H. Behnke and F. Korte [16] have introduced already in 1935 a classification of the boundary points of a domain into "supporting points" ("Stützpunkte") and "flat points" ("Flachpunkte").

Given a domain $D$ and a subdomain $G \subset D$, then the envelopes of holomorphy $E(G)$ and $E(D)$ may coincide, or not, depending upon $G$.

If $z^{(0)}$ is a boundary point of $D$ such that it is also a boundary point of all subdomains of $G$ for which $E(G)=E(D)$, then $z^{(0)}$ is called a "supporting point," otherwise a "flat point." In other words $z^{(0)}$ is a flat point if and only if for sufficiently small neighborhoods $N\left(z^{(0)}\right)$ we have $E\left(D-N\left(z^{(0)}\right)\right)=E(D)$.

For $n=2$ and domains of holomorphy with a sufficiently smooth boundary $\mathrm{H}$. Behnke and K. Stein have given a characterization of the supporting points and flat points by means of Levi's differential expression (H. Behnke and $\mathrm{K}$. Stein [3]).

Theorem. The Silov boundary of a domain D coincides exactly with the set of "supporting boundary points" of $D$. 
Proof. Let $z^{(0)}$ be a flat point, then there exists a neighborhood $N\left(z^{(0)}\right)$ such that $E\left(D-N\left(z^{(0)}\right)\right)=E(D)$. Then, according to Theorem 6.6 we have

$$
S(D)=S(E(D))=S\left(E\left(D-N\left(z^{(0)}\right)\right)\right) \subset \partial\left(D-N\left(z^{(0)}\right)\right)
$$

Hence

$$
S(D) \cap N\left(z^{(0)}\right)=0 .
$$

Therefore $z^{(0)}$ does not belong to $S(D)$. On the other hand a point $z^{(1)}$ on the Šilov boundary cannot be a flat point, because there exist functions holomorphic in $D$ assuming their maximum arbitrarily close to the given point $z^{(1)}$. Hence there exist constants $a$ and functions $F(z)$, holomorphic in $D$ such that $F(z)=a$ for some $z \in D$, but $f(z) \neq a$ outside an arbitrary small neighborhood of $z^{(1)}$. This implies that for no neighborhood $N\left(z^{(1)}\right)$ of $z^{(1)}$ we have $E(D)=E\left(D-N\left(z^{(1)}\right)\right)$. Hence $z^{(1)}$ is a supporting point.

7. The Dirichlet problem for more general domains.

7.1. We now return to the question of the generalized Dirichlet problem for plurisubharmonic functions.

As we have seen above, the (generalized) Dirichlet problem is possible for the upper envelope $\phi(z)$ of all functions that are holomorphic in $\bar{D}$ and smaller or equal the prescribed boundary values if:

(a) $D$ is strictly pseudo-convex and bounded and the values $b(z)$ are prescribed in the Silov boundary $S(D)$;

(b) $D$ is an analytic polyhedron and the values $b(z)$ are prescribed on $S(D)$.

We want to investigate the problem for more general domains.

Two cases have to be distinguished:

(1) The case where $D$ is a domain of holomorphy.

(2) $D$ is not a domain of holomorphy.

THEOREM. For bounded domains of holomorphy of the type $D=\{z \mid V(z)<0\}$, $V(z)$ plurisubharmonic in a neighborhood of $D$, the Silov boundary with respect to the class of moduli of holomorphic functions and the Silov boundary with respect to the class of functions that are plurisubharmonic in a neighborhood of $D$ coincide.

(Under the Silov boundary of $D$ with respect to a class of functions $\mathbb{R}$ we understand the smallest closed subset $S_{\mathfrak{R}}$ of $\partial D$ such that if $f \in \mathfrak{R}$ and $f \leqq M$ on $S_{\mathfrak{R}}$, then $f \leqq M$ in $\bar{D}$.) This follows immediately from Lemma 6.2 and from 6.11 .

The theorem does not hold for certain domains that are not domains of holomorphy. The example given in Bremermann [11] shows that there exists a domain $D$ and a function $V(z)$ plurisubharmonic in $D$ such that $V(z)=0$ on $S(D)$ but $V(z)>0$ in certain points of $D$.

Thus Case 2 cannot be reduced to Case 1 (as it could be in the determination of the (ordinary) Silov boundaries). We treat in the following only Case 1. 
7.2. Let $\mathbb{R}(D, b(z))$ be the class of functions that are plurisubharmonic in a neighborhood of $D$ and smaller or equal the boundary values $b(z)$ wherever these are prescribed.

Theorem. Let $D$ be a bounded pseudo-convex domain of the form $D$ $=\{z \mid V(z)<0\}, V(z)$ plurisubharmonic and continuous in a neighborhood of $D$. Then the (generalized) Dirichlet problem is possible for the upper envelope $\phi(z)$ of $\mathfrak{R}(D, b(z))$ and arbitrary continuous boundary values $b(z)$ if and only if the boundary values $b(z)$ are prescribed on and only on the Silov boundary $S(D)$ of $D$.

(1) $\phi(z)$ cannot be larger on $\partial D$ than its maximum on $S(D)$. Hence $\phi(z)$ cannot assume any values on $\partial D-S(D)$ that are larger than the maximum of the values prescribed on $S(D)$. Hence it is clear that one cannot prescribe arbitrary boundary values on a subset of $\partial D$ that is not contained in $S(D)$.

(2) On the other hand we have seen in 6.10 and 6.11 that under the conditions of our theorem there exists for any $z_{0} \in S(D)$ and arbitrarily small $r$, a function $f$, holomorphic in $\bar{D}$ that assumes its maximum with respect to $\bar{D}$ in $\partial D \cap\left\{z \mid\left\|z-z_{0}\right\|<r\right\}$ and is smaller than its maximum in $\partial D-\left\{z \mid\left\|z-z_{0}\right\|<r\right\}$.

Hence if the values $b(z)$ are prescribed such that a neighborhood of a point $z_{0} \in S(D)$ is left out, then we have an $r$ and a function $f$ such that $|f|$ is smaller or equal to $M=\max b(z)$ in $\partial D-\left\{z|| \mid z-z_{0} \|<r\right\}$, but $|f|>M$ in some points of $\bar{D} \cap\left\{z \mid\left\|z-z_{0}\right\|<r\right\}$. Then the function

$$
|g(z)|=M(|f| / M)^{n}
$$

is for $n$ large enough, larger than any given number $N$ in some points of $\bar{D} \cap\left\{z \mid\left\|z-z_{0}\right\|<r\right\}$, but still smaller or equal to $b(z)$ in $\partial D-\left\{z \mid\left\|z-z_{0}\right\|<r\right\}$.

The function $|g(z)|$ is in particular plurisubharmonic in $D$, hence in the class $\mathfrak{R}(D, b(z))$.

It shows that the class $R(D, b(z))$ is not bounded from above in $\bar{D}$ and hence the upper envelope $\phi(z)$ does not exist.

Points (1) and (2) combined show that the (generalized) Dirichlet problem is possible for $D$ only if the values $b(z)$ are prescribed exactly on $S(D)$. This proves half of our theorem.

(3) The converse, that $\phi(z)$ exists and assumes the given values $b(z)$ (in the sense that $\left.\lim \sup _{z^{\prime} \rightarrow z} \phi\left(z^{\prime}\right)=b(z)\right)$, follows from 6.11 in almost exactly the same way as in 4.1 .

REMARK. Theorems 4.1 and 5.3 now follow from Theorems 7.2, 6.3, and 6.4. We have nevertheless given direct proofs in 4.1 and 5.3 as they are simpler than the argument involving the Silov boundary and do not make use (as 6.3 and 7.2 do) of the rather deep Lemma 6.2.

7.3. Corollary For ONE COMPleX VARIABle. Let $D$ be a bounded domain in the plane of one complex variable, $D=\{z \mid V(z)<0\}$, where $V(z)$ is continuous and subharmonic in a neighborhood of $D$. Then the Dirichlet problem (for harmonic functions) is possible for $D$. 
This follows from 7.2 if we know that $S(D)=\partial D$ (because 7.2 holds in particular for $n=1$, and because in this case the upper envelope $\phi(z)$ is harmonic, which is well known. The "Dirichlet problem is possible" means that $\phi(z)$ actually assumes the given boundary values. $S(D)=\partial D$ does not hold for instance if $D$ is the unit disc minus the origin).

If $D$ is of the form described above, then $D$ is relative compact in the larger domain $D_{\boldsymbol{\epsilon}}=\{z \mid V(z)<\epsilon\}$, for some $\epsilon>0$, and $D$ is relatively simply connected with respect to $D_{\epsilon}$ (compare Bremermann [14]).

Let $z_{0}$ be a point on the boundary, then the function $1 /\left(z-z_{0}\right)$ has a pole at $z_{0}$ and is otherwise holomorphic in $D$. However, because $D$ is relatively simply connected and compact in $D_{\epsilon}$, the pole can be moved to $z_{1} \in D_{\epsilon}-\bar{D}$ such that $1 /\left(z-z_{1}\right)$ has its maximum with respect to $\bar{D}$ at $z_{0}$. It follows that the Silov boundary is the whole boundary.

7.4. Properties of the plurisubharmonic solution. The plurisubharmonic solution as described above has the following properties:

(1) The solution $\phi(z)$ for values on $S(D)$ is unique.

(2) If the values $b(z)$ belong to the real part $h(z)$ of a holomorphic function, then $h(z)=b(z)$. This property follows from the fact that the pluriharmonic functions form a subclass of the plurisubharmonic functions, and that every $\psi(z) \in \mathfrak{R}(D, b(z))$ is majorized by $h(z)$. (Conditions that $b(z)$ belongs to a holomorphic function have been given by Severi [31] and Lewy [20], while Rizza [30] has given an integral formula expressing a pluriharmonic function by its values on the boundary of a pseudo-convex domain.)

(3) $\phi(z)$ is pseudo-conformally invariant. More precisely: Let $z^{*}(z)$ be a one to one pseudo-conformal mapping of a neighborhood of $D$ onto a neighborhood of $D^{*}$. Let $b^{*}\left(z^{*}\right)=b\left(z\left(z^{*}\right)\right)$. If then $\phi(z)$ is the solution of $b(z)$ on $S(D)$, then $\phi\left(z\left(z^{*}\right)\right)$ is the solution of $b^{*}\left(z^{*}\right)$ on $S\left(D^{*}\right)$.

This follows from the fact that under these conditions $S(D)$ is transformed into $S\left(D^{*}\right)$ and, because every function pseudo-convex in $D$ is transformed into a pseudo-convex function in $D^{*}$ and vice versa, the class $\mathfrak{R}(D, b(z))$ is transformed into $\mathfrak{R}\left(D^{*}, b^{*}\left(z^{*}\right)\right)$.

(4) $\phi(z)$ is subadditive, but in general not additive. That means: Let

$$
\begin{aligned}
& \phi_{1}(z) \text { be the solution of } b_{1}(z), \\
& \phi_{2}(z) \text { the solution of } b_{2}(z), \\
& \phi(z) \text { the solution of } b_{1}(z)+b_{2}(z),
\end{aligned}
$$

then

$$
\phi_{1}(z)+\phi_{2}(z) \leqq \phi(z) \text { in } \bar{D},
$$

and it actually happens for certain domains and certain boundary values that equality does not hold.

If $\phi_{1}(z)$ assumes the values $b_{1}(z)$ and $\phi_{2}(z)$ the values $b_{2}(z)$, then $\phi_{1}(z)$ 
$+\phi_{2}(z)$ will assume the values $b_{1}(z)+b_{2}(z)$. However, while $\phi_{1}(z)$ is the upper envelope of the plurisubharmonic functions assuming $b_{1}$ and $\phi_{2}$ correspondingly, we cannot conclude that $\phi_{1}+\phi_{2}$ is also the upper envelope of

$$
\mathfrak{R}\left(D, b_{1}(z)+b_{2}(z)\right) \text {. }
$$

Hence the above inequality follows, and examples that the equality actually breaks down can be given easily in the form of tube-domains.

7.5. REMARK. The fact that $\phi(z)$ is subadditive but not additive prevents the representation of $\phi(z)$ in the form of an integral over the boundary values (analog to the solution of the Dirichlet problem for harmonic functions by harmonic measures).

Such an integral representation is convenient for the actual calculation of $\phi(z)$ for a specific problem and to derive estimates. The connection with the theory of envelopes of holomorphy, which is treated in the following section, provides us with a substitute that serves both these purposes.

7.6. The plurisuperharmonic solution. If we would have taken the plurisuperharmonic functions larger or equal $b(z)$ on $S(D)$ and the lower envelope we would have obtained a plurisuperharmonic solution $\Phi(z)$. Obviously $\Phi(z)$ exists if and only if $\phi(z)$ exists. We have

$$
\phi(z)=\Phi(z) \text { on } S(D)
$$

and

$$
\phi(z) \leqq \Phi(z) \text { in } D .
$$

If equality holds in one interior point of $D$, then it holds for all points of $D$ and $\phi(z)=\Phi(z)$ is pluriharmonic. This follows because if $\Phi(z)$ is plurisuperharmonic, then $-\Phi(z)$ is plurisubharmonic, hence $\phi(z)-\Phi(z)$ is plurisubharmonic, hence takes its maximum on $S(D)$. On $S(D)$, however, we have $\phi(z)-\Phi(z)=0$, therefore $\phi(z)<\Phi(z)$ in $D$. If equality holds at an interior point of $D$, then the plurisubharmonic function $\phi(z)-\Phi(z)$ takes its maximum at an interior point and hence must be a constant. Also, then $\phi(z)=\Phi(z)$ is plurisub- and superharmonic, hence pluriharmonic.

In particular the harmonic solution of the boundary value problem exists (this follows as in 4.1). (If $S(D) \neq \partial D$ we can prescribe $\phi(z)$ on all of $\partial D$ as boundary values.) We have

$$
\phi(z) \leqq h(z) \leqq \Phi(z) \text { in } \bar{D},
$$

and if equality holds in one interior point of $D$, then in all points of $D$.

$h(z)$ is not invariant under pseudo-conformal transformations, but $\phi(z)$ and $\Phi(z)$ are. Hence we have:

Lemma. Let $h(z), \phi(z)$ and $\Phi(z)$ be the harmonic, plurisub-and plurisuperharmonic solutions of the boundary values $b(z)$ on $S(D)$. Let $h^{*}\left(z^{*}\right)$ be the har- 
monic solution of the corresponding boundary value problem for $D^{*}$, where $D^{*}$ is the image of $D$ under the one-to-one pseudo-conformal transformation $z^{*}(z)$ of a neighborhood of $D$. Then, in general,

$$
h(z) \neq h^{*}\left(z^{*}(z)\right),
$$

however

$$
\phi(z) \leqq h^{*}\left(z^{*}(z)\right) \leqq \Phi(z) .
$$

Corollary. Let $D=\{z \mid V(z)<0\}$ and $D^{*}$ be the image of $D$ under a one-toone pseudo-conformal transformation $z^{*}(z)$ of a neighborhood of $D$. Let $h(z)$ be harmonic in $D, h^{*}\left(z^{*}\right)$ harmonic in $D^{*}$ and $h(z)=h^{*}\left(z^{*}(z)\right)$ on $\partial D$, then $\left|h(z)-h^{*}\left(z^{*}(z)\right)\right|<\Phi(z)-\phi(z)$, where $\phi(z)$ and $\Phi(z)$ are the plurisub-and plurisuperharmonic solutions of the restriction of $h(z)$ to $S(D)$.

7.7. Let $D \subset C^{2}$ be a domain such that the distinguished boundary surface and the Silov boundary coincide. Let $\phi(z)$ be the plurisubharmonic solution, $\Phi(z)$ the plurisuperharmonic solution and $B(z)$ a solution of any of the possible Bergman extended classes. Then we have

$$
\phi(z) \leqq B(z) \leqq \Phi(z) \text { in } \bar{D} .
$$

$B(z)$ is either a harmonic function, then the inequality holds, or $B(z)$ is defined on $\partial D$ by decomposing $\partial D$ into segments of analytic surfaces whose boundaries lie on $\delta D$. Then for each segment the ordinary harmonic boundary value problem is solved. If now $\phi(z)$ is the plurisubharmonic solution, then its restriction to the segments is subharmonic and $B(z)=\phi(z)$ on $\delta D$. Hence by the fundamental property of the subharmonic functions $B(z) \geqq \phi(z)$ holds on each segment and hence on $\partial D . B(z)$ is then defined in $D$ by decomposing $D$ into a two-parameter family of analytic surfaces with boundaries on $\partial D$ and defining $B(z)$ as the harmonic function that assumes the values in the intersection with $\partial D$. Therefore the same conclusion as above applies and we have shown

$$
\phi(z) \leqq B(z) \text { in } \bar{D},
$$

and analogously $B(z) \leqq \Phi(z)$ follows.

REMARK. For a special case the existence of $\phi(z)$ and $\Phi(z)$ and the above inequality have already been proved by S. Bergman [3].

7.8. Lemma. If $\left|b_{1}(z)-b_{2}(z)\right|<\epsilon$ for $z \in S(D)$, then, if $\phi_{1}(z)$ and $\phi_{2}(z)$ are the corresponding solutions, then

$$
\left|\phi_{1}(z)-\phi_{2}(z)\right|<\epsilon \text { for } z \in \bar{D} \text {. }
$$

If $\left|b_{1}(z)-b_{2}(z)\right|<\epsilon$, then $b_{1}(z)<b_{2}(z)+\epsilon$, and $\phi_{1}(z) \in \mathfrak{R}\left(D, b_{2}(z)+\epsilon\right)$. The upper envelope of $\mathfrak{R}\left(D, b_{2}(z)+\epsilon\right)$ is $\phi_{2}(z)+\epsilon$. Hence we have

$$
\phi_{1}(z) \leqq \phi_{2}(z)+\epsilon,
$$


and for symmetry reasons also

$$
\phi_{2}(z) \leqq \phi_{1}(z)+\epsilon,
$$

hence

$$
\left|\phi_{1}(z)-\phi_{2}(z)\right|<\epsilon .
$$

7.9. Extremal property of $\phi(z)$. If $\phi(z)$ is the solution of a boundary value problem and if $\phi(z)$ is $\left(C^{2}\right)$, then we have at any point $z_{0}$ in $D: \bar{\partial} \partial \phi=0$ for some direction.

Suppose we would have $\bar{\partial} \partial \phi>\sigma$ at $z_{0} \in D$. Then $\phi(z)-\epsilon u(z)\left\|z-z_{0}\right\|^{2}$, where $u(z)$ is as in 6.8 and $\epsilon$ sufficiently small, would be still plurisubharmonic in $\bar{D}$, and for some $\delta>0$

$$
\tilde{\phi}(z)=\phi(z)-\epsilon u(z)\left\|z-z_{0}\right\|^{2}+\delta
$$

would be on $S(D)$ smaller or equal the boundary values $b(z)$ that $\phi(z)$ is assumed to solve. Hence $\tilde{\phi}(z) \in \mathfrak{R}(D, b(z))$, but

$$
\tilde{\phi}\left(z_{0}\right)>\phi\left(z_{0}\right),
$$

hence $\phi(z)$ could not be the upper envelope of $\&(D, b(z))$. This is a contradiction. $\bar{\partial} \partial \phi>0$ for all directions is not possible.

8. Connection of $\phi(z)$ with the envelopes of holomorphy of Hartogs domains. The plurisubharmonic solution $\phi(z)$ as considered in the previous sections is closely connected with the envelopes of holomorphy of Hartogs domains. As the author has shown in a recent paper (Bremermann [13]) envelopes of holomorphy can be calculated explicitly. And thus we are provided with a method actually to calculate $\phi(z)$.

8.1. Let $D=\{z \mid V(z)<0\}$, satisfying the usual conditions. Let $b(z)$ be continuous boundary values prescribed on the Silov boundary $S(D)$.

It is inconvenient to have the boundary values only on $S(D)$. We continue them to a function $\phi^{*}(z)$ defined in all of $\bar{D}$ :

$$
\begin{aligned}
& \phi^{*}(z)=b(z) \text { on } S(D), \\
& \phi^{*}(z)=\max _{z \in S(D)} b(z) \text { on } \bar{D}-S(D) .
\end{aligned}
$$

Then we have $\phi(z)=\phi^{*}(z)$ on $S(D)$ and

$$
\phi(z) \leqq \phi^{*}(z) \text { in } \bar{D} .
$$

We now consider the pointset

$$
H=\left\{(z, w)|z \in \bar{D},| w \mid<e^{-\phi^{*}(z)}\right\} .
$$

In the following we will determine the envelope of holomorphy of this point set, which is closely related to the "Hartogs domains." 
8.2. In $[10 ; 12]$ the author has proved the following lemma:

Let $G$ be a domain in the space of $s$ complex variables $\xi_{1} \cdots \xi_{8}$. Let

$$
d_{a, a}(\zeta)=\sup r \ni\left\{\zeta^{\prime}\left|\zeta^{\prime}=\zeta+\lambda a,\right| \lambda \mid<r\right\} \subset G,
$$

in other words $d_{a, G}(\zeta)$ is the distance of the point $\zeta$ from the boundary of $G$ in the (complex) direction $a$. Let $\Delta$ be a domain on an analytic plane

$$
\left\{\zeta \mid \zeta=\zeta_{0}+\tau b\right\}
$$

let $\bar{\Delta} \subset G$. Let $h(\zeta)$ be harmonic (as a function of $\tau$ ) in $\Delta$ and continuous in $\bar{\Delta}$.

Let

$$
\mu \leqq \min _{\zeta \in \partial \Delta} d_{a, G}(\zeta) e^{h(\zeta)}
$$

Then any function holomorphic in $G$ can be continued holomorphically into the point set

$$
\left\{\zeta\left|\zeta=\zeta^{\prime}+\lambda a,\right| \lambda \mid<\mu e^{-h\left(\zeta^{\prime}\right)}, \zeta^{\prime} \in \Delta\right\} .
$$

REMARK. In [12] the lemma is stated only under the assumption that $\chi(z)=e^{h(z)+i h^{*}(z)}$, where $h^{*}(z)$ is a conjugate harmonic function of $h(z)$, is holomorphic and hence single valued in $\Delta$. In the proof is used only that $|\chi(z)|$ is single valued, but not $\chi(z)$ itself. Hence we can state the lemma in the above form.

In the following we apply this lemma to $H$. As direction $a$ we choose the $w$-plane, $a=(0, \cdots, 0,1) . H$ is not an open set. If we speak of a function "holomorphic in $H$ " we mean that it is holomorphic in an open set $N(H)$ containing $H$.

Then $d_{a, N(H)}(z, 0) \geqq e^{-\phi^{*}(z)}$ in $\bar{D}$.

Let now $P$ be any one dimensional analytic plane of the form

$$
P=\left\{z \mid z=z_{0}+\sigma c\right\},
$$

and $\Delta=D \cap P$. We observe that

$$
\Delta=\{z \mid \tilde{V}(z)<0\}
$$

if $\tilde{V}(z)$ is the restriction of $V(z)$ to $P$. Hence, according to Corollary 7.3, the ordinary Dirichlet problem for harmonic functions and continuous boundary values is solvable for $\Delta$.

The restriction of $\phi^{*}(z)$ to $\partial D$ will in general not be continuous. We therefore approximate by continuous functions $b^{*}(z)$ such that

$$
b^{*}(z) \geqq \phi^{*}(z) \text { on } \partial D \text {. }
$$

Then the boundary value problem

$$
h(z)=b^{*}(z) \text { on } \partial \Delta
$$


and $h(z)$ harmonic in $\Delta$ is solvable according to the preceding remarks. We have

$$
d_{a, N(H)}(z, 0) \geqq e^{-\phi^{*}(z)} \geqq e^{-h(z)} \text { on } \partial \Delta .
$$

Hence $\min _{\Delta} d_{a, N(H)}(z, 0) e^{h(z)} \geqq 1$. Hence any function holomorphic in $H$ can be continued holomorphically into the point set

$$
\left\{(z, w)|z \in \Delta,| w \mid<e^{-h(z)}\right\} .
$$

By repeating this for every intersection $P \cap D$ we obtain:

LEMma. Every function holomorphic in $H$ is holomorphically continuable in the point set:

$$
\left\{(z, w)|z \in \bar{D},| w \mid<e^{-\psi(z)}\right\},
$$

where $\psi(z)$ is the lower envelope of all the functions $h_{\Delta_{P}}(z)$, where $h_{\Delta_{P}}(z)$ is the harmonic solution of the boundary value problem

$$
h_{\Delta_{P}}(z)=b^{*}(z) \text { on } \partial \Delta_{P},
$$

and where $\Delta_{P}$ is the intersection of $D$ with an arbitrary one-dimensional analytic plane $P$.

We note that $\psi(z)$ is continuous and $\psi(z) \geqq \phi(z)$ in $\bar{D}$.

8.3. In order to obtain the envelope of holomorphy of $H$ we continue as follows:

Let $\Gamma$ be a disc on an arbitrary one-dimensional analytic plane $P$. Then we take the orthogonal complement $O(P)$ of $P$ and all discs $\Gamma^{*}$ parallel to $\Gamma$, of same radius as $\Gamma$ with centers in $O(P)$ that lie in $\bar{D}$. For these discs we solve the boundary value problem

$$
h_{\Gamma^{*}}(z)=\psi(z) \text { on } \partial \Gamma^{*}
$$

and replace $\psi(z)$ in $\bar{\Gamma}^{*}$ by inf $\left\{\psi(z), h_{\Gamma^{*}}(z)\right\}$. Doing this for all $\Gamma^{*}$ we obtain a new function $\psi_{\Gamma}(z)$. We note that $\psi_{\Gamma}(z)$ is again continuous.

From our lemma follows that all functions holomorphic in $H^{*}$ can be continued holomorphically into

$$
\left\{(z, w)|z \in \bar{D},| w \mid<e^{-\psi_{\Gamma}(z)}\right\} .
$$

This procedure is repeated for all discs $\Gamma_{\nu}$ where $\Gamma_{\nu}$ is a denumerable dense subset in the set of all discs in $D$ (such a subset has explicitly been described in Bremermann [13]), applying it always to the previously generated function $\psi_{\Gamma_{v-1}}(z)$.

Because $\phi(z)$ is plurisubharmonic and because we had $\psi(z) \geqq \phi(z)$ to begin with, each $\psi_{\Gamma_{v}}(z)$ has the property that

$$
\psi_{\Gamma_{\nu}}(z) \geqq \phi(z) .
$$


Also, the $\psi_{\Gamma_{\nu}}(z)$ constitute a monotone sequence, therefore a limit function $\psi_{0}(z)$ exists, and it is

$$
\psi_{0}(z) \geqq \phi(z) \text { in } \bar{D} .
$$

Also $\psi_{0}(z)$ is plurisubharmonic, because from the construction and from being the limit function it follows that we have $\psi_{0}(z) \leqq h_{\gamma}(z)$ in $\gamma$ for any disc $\gamma \in D$ and for any harmonic function $h_{\gamma}(z)$ such that $h_{\gamma}(z) \geqq \psi_{0}(z)$ on $\partial \gamma$. But this property implies that $\psi_{0}(z)$ is plurisubharmonic in $D$.

We had approximated the function $\phi^{*}(z)$ by a continuous function $b^{*}(z)$ on $\partial D$. Letting $b^{*}(z)$ tend to $\phi^{*}(z)$ monotonously from above, the corresponding functions $\psi_{0}(z)$ will tend to a limit function $\psi_{00}(z)$ that is plurisubharmonic in $D$ and assumes the values $b(z)$ on $S(D)$. For $\psi_{00}(z)$ we have

$$
\psi_{00}(z) \geqq \phi(z) \text { in } \bar{D} \text {. }
$$

On the other hand $\phi(z)$ is the upper envelope of all plurisubharmonic functions in $D$ assuming the values $b(z)$ on $S(D)$, therefore

$$
\psi_{00}(z)=\phi(z)
$$

holds.

Thus any function holomorphic in $H$ can be continued holomorphically into the set

$$
H^{* *}=\left\{(z, w)|z \in D,| w \mid<e^{-\phi(z)}\right\} .
$$

On the other hand this domain is a domain of holomorphy; this follows easily from Oka's theorem:

We can write $H^{* *}$ in the form

$$
H^{* *}=\{(z, w)|V(z)<0, \log | w \mid+\phi(z)<0\} .
$$

If we introduce $\tilde{V}(z, w)=\sup \{V(z), \log |w|+\phi(z)\}$ we can write

$$
H^{* *}=\{(z, w) \mid \tilde{V}(z, w)<0\} .
$$

$\tilde{V}(z, w)$ is plurisubharmonic in a neighborhood of $H^{* *}$, hence $H^{* *}$ is pseudoconvex, and hence by Oka's theorem a domain of holomorphy (Oka [27] and [28], see also Bremermann [9] and Norguet [26]).

From this and from the fact that any function holomorphic in $H$ can be continued into $H^{* *}$ follows that $H^{* *}$ is the largest open set contained in the envelope of holomorphy of $H$.

8.4. Summarizing the results of this section we obtain:

Theorem. Let $D=\{z \mid V(z)<0\}$ be a bounded domain such that $V(z)$ is plurisubharmonic in a neighborhood of $D$. Let $b(z)$ be continuous boundary values prescribed on the Silov boundary $S(D)$. Let $\phi(z)$ be the plurisubharmonic solution of the Dirichlet problem. Then the point set 


$$
\left\{(z, w)|z \in \bar{D},| w \mid<e^{-\phi(z)}\right\},
$$

is the envelope of holomorphy of the point set

$$
\left\{(z, w)|z \in \bar{D},| w \mid<e^{-\phi^{*}(z)}\right\},
$$

where

$$
\begin{aligned}
& \phi^{*}(z)=b(z) \text { on } S(D), \\
& \phi^{*}(z)=\max _{z \in S(D)} b(z) \text { on } \bar{D}-S(D) .
\end{aligned}
$$

Corollary. $\phi(z)$ can be computed.

In [13] we have shown that if both $G$ and the envelope of holomorphy $E(G)$ are schlicht (which is the case here) then $E(G)$ can be determined from $G$ by a constructive process which with a finite number of calculations approximates $E(G)$ to arbitrary accuracy.

REMARK. We have for the sake of simplicity conducted the proof of the theorem not constructively. However the process of holomorphic continuation from $H$ to $H^{* *}$ can be discretizised in a similar way as this has been done for the general construction of envelopes of holomorphy in [13]. Such an adaptation of the process to construct $E(H)$ to the special form of $H$ will simplify the computation.

\section{Generalizations, examples, applications.}

9.1. Upper semi-continuous boundary values. The condition that the prescribed boundary values be continuous can be relaxed. If $b(z)$ is prescribed on $S(D)$ and only upper semi-continuous, we can approximate from above by a monotone sequence of continuous functions $b_{\nu}(z)$. Thus we obtain a monotone sequence $\phi_{\nu}(z)$ that converges towards a limit function $\phi(z) . \phi(z)$ is plurisubharmonic and wherever $\lim \sup _{z \rightarrow z_{0}} b(z)=b\left(z^{0}\right)$ we have $\lim \sup _{z \rightarrow z_{0}} \phi(z)$ $=b\left(z_{0}\right)$.

9.2. Boundary values of $\log |f|, f$ holomorphic. Nevanlinna theory. The key to applications as in the theory of Nevanlinna is the solution of the boundary values that are induced by $\log |f|$, where $f$ is holomorphic in a neighborhood of $D$ such that $\phi(z)$ is finite in $D$. (Compare Nevanlinna [25], Bergman $[5 ; 6]$.)

If $f$ has no zeros in $\bar{D}$, then $\log |f|$ itself is the solution, but if $f$ becomes zero somewhere in $D$, then, other as in the case of only one variable, the zeros of $f$ will also intersect the boundary of $D . \log |f|$ itself is again a plurisubharmonic solution of this boundary value problem, but $\log |f|$ is not finite in $D$. We conjecture, that in general if $D$ is a bounded pseudo-convex domain, $f$ holomorphic in a neighborhood of $D, b(z)=\log |f|$ on $S(D)$, then this boundary value problem can be solved by a plurisubharmonic function $\phi(z)$ that is finite throughout $D$.

9.3. Stein manifolds. We have developed the previous sections for sim- 
plicity's sake for schlicht domains only. However, complex manifolds have become increasingly important. Not all complex manifolds are suitable for a theory of functions but it has been shown that for Stein manifolds most of the results from schlicht domains and from Riemann surfaces in one variable are valid. This is true for our results also.

A Stein manifold of complex dimension $n$ is a complex manifold with countable base satisfying the following three axioms.

(1) For every point $q \in \mathfrak{M}^{n}$ there exist $n$ functions $h_{1}(p) \cdots h_{n}(p)$ holomorphic in $\mathfrak{M}^{n}$ that are local coordinates in a neighborhood of $q$.

(2) To every two different points $p, q$ there exists a function $f$ holomorphic in $\mathfrak{M}^{n}$ that separates $p$ and $q$, that is $f(p) \neq f(q)$.

(3) $\mathfrak{M}^{n}$ is the limit of a sequence of analytic polyhedra relative compact in $\mathfrak{M}^{n}$.

We will examine now the main points of the previous sections.

First of all, plurisubharmonic functions are defined on Stein manifolds because the property of a function to be plurisubharmonic is a local property and it is invariant under pseudo-conformal transformations. All the main properties remain true. Pseudo-convex domains can be defined as in the schlicht case by means of plurisubharmonic functions.

In 4.1 and 6.8 we make use of the euclidean distance $\left\|z-z_{0}\right\|$. If we have a relative compact subdomain $D$ of a Stein manifold, then we can find finitely many functions $f_{1} \cdots f_{s}$ such that for any pair of points $p, q \in D$ at least one of the functions has different values at $p$ and at $q$. Then $\left|f_{1}(p)-f_{1}(q)\right|^{2}+\cdots$ $+\left|f_{s}(p)-f_{s}(q)\right|^{2}$ has the property that the sum is zero if and only if $p=q$. Also, if a function $V(p)$ is strictly plurisubharmonic in a neighborhood of $D$, then $V(p)-\epsilon \sum_{j=1}^{s}\left|f_{j}(p)-f_{j}(q)\right|^{2}$ will still be plurisubharmonic for $\epsilon$ sufficiently small. But these are exactly the properties of $\left\|z-z_{0}\right\|$ that were used. Hence we can replace $\left\|z-z_{0}\right\|$ by $\sum\left|f_{j}(p)-f_{j}(q)\right|^{2}$.

Finally we made important use of Lemma 6.2. But this lemma has been proved for Stein manifolds also (Bremermann [14]).

Difficulties arise where the euclidean distance functions $d_{D}(z)$ or $d_{a, D}(z)$ are used. Here modifications are necessary. Hence we can state:

The results of $\S \S 3-7$ and 9.1 are valid for relative compact pseudo-convex subdomains of Stein manifolds also. The extension of $\S 8$ requires a few modifications.

9.4. Banach spaces of infinite dimension. Some of the results of the preceding sections (though at the present moment not all) can be extended to general complex Banach spaces.

Plurisubharmonic functions and pseudo-convex domains are defined for infinite dimension as for finite dimension. (For details compare Bremermann [12].)

Let us consider the case of strictly pseudo-convex domains. Let $D$ be a bounded domain in our complex Banach space $B_{c}$. Let $D=\{z \mid V(z)<0\}$, 
where $V(z)$ is plurisubharmonic in a neighborhood of $D$. Let $V(z)$ be such that

$$
\left.\frac{\partial^{2} V\left(z_{0}+\lambda a\right)}{\partial \lambda \partial \bar{\lambda}}\right|_{\lambda=0} \geqq \epsilon>\sigma
$$

for $\epsilon$ fixed and for all $z_{0}$ in $\bar{D}$ and for all complex directions $a \in B_{c}$. (This is the substitute for $\bar{\partial} \partial V>0$ in the finite case.)

Let $B_{c}$ possess a norm $N(z)$ which is $\left(C^{2}\right)$. Let the derivatives of $N(z+\lambda a)$ be bounded. Then

$$
V(z)-\delta N^{2}\left(z-z_{0}\right)
$$

will still be a plurisubharmonic function in $\bar{D}$ for $\delta$ sufficiently small.

Let $z_{0}$ be a boundary point of $D$. Then

$$
V^{*}(z)=V(z)-\delta N^{2}\left(z-z_{0}\right)
$$

has the property to be plurisubharmonic in $\bar{D}, V^{*}\left(z_{0}\right)=0$ and $V^{*}(z)<0$ in $\bar{D}-\left\{z_{0}\right\}$.

From here it follows as in 4.1 that we can solve the Dirichlet problem for continuous boundary values on $\partial D$. Thus we have shown:

Theorem. Let $D=\{z \mid V(z)<0\}$ be a bounded domain in a complex Banach space $B_{c}, V(z)$ plurisubharmonic in a neighborhood of $D$. Let $B_{c}$ possess a $\left(C^{2}\right)$ norm $N(z)$ and let the second derivatives of $N\left(z_{0}+\lambda a\right)$ be uniformly bounded in $D$ for every $z_{0}$ and $a \in B_{c}$ with $N(a)=1$. Let

$$
\left.\frac{\partial^{2} V\left(z_{0}+\lambda a\right)}{\partial \lambda \partial \bar{\lambda}}\right|_{\lambda=0} \geqq \epsilon>0
$$

for fixed $\epsilon$ and for all $z_{0}$ in $\bar{D}$ and $a \in B_{c}$ with $N(a)=1$. Then the Dirichlet problem is solvable, for continuous boundary values prescribed in $\partial D$, by plurisubharmonic functions (as in 4.1).

9.5. Tube-domains, examples. We now return for the sake of simplicity to finite dimension. A tube domain is a domain of the form

$$
T_{B}=\{z \mid x \in B, y \text { arbitrary }\},
$$

where $z_{j}=x_{j}+i y_{j}, x=\left(x_{1}, \cdots, x_{n}\right), y=\left(y_{1}, \cdots, y_{n}\right)$ and $B$ a domain in the (real) $x$-space. is:

Tube domains serve frequently as examples, their fundamental property

$A$ tube domain $T_{B}$ is pseudo-convex if and only if $B$ is convex. (Compare Bremermann [7].)

In the following we will see that for tube domains not only the pseudoconvexity but the Šilov boundary, too, has a simple interpretation.

Let $B$ be convex and bounded. Let $x^{0}$ be a point where $B$ is strictly 
convex, that means there exists a supporting plane or hyperplane not intersecting $\bar{B}-\left\{x^{0}\right\}$.

Then there exists also a sphere

$$
\left\{x \mid\left(x_{1}-a_{1}\right)^{2}+\cdots+\left(x_{n}-a_{n}\right)^{2}=r^{2}\right\}
$$

that goes through $x^{0}$ and does not intersect $\bar{B}$. By replacing $x_{1}, \cdots, x_{n}$ by $z_{1}, \cdots, z_{n}$ we obtain an analytic surface:

$$
\mathscr{F}=\left\{z \mid\left(z_{1}-a_{1}\right)^{2}+\cdots+\left(z_{n}-a_{n}\right)^{2}=r^{2}\right\} .
$$

Now $\left(z_{j}-a_{j}\right)^{2}=\left(x_{j}-a_{j}\right)^{2}+2 i\left(x_{j}-a_{j}\right) y_{j}-y_{j}^{2}$, hence if $z \in \mathcal{F}$, then

$$
\sum_{j=1}^{n}\left(x_{j}-a_{j}\right)^{2}=r^{2}+\sum_{j=1}^{n} y_{j}^{2}
$$

therefore if $y_{1}, \cdots, y_{n} \neq(0, \cdots, 0)$, then $x \notin B$, hence $z \notin T_{B}$.

Thus the function

$$
f(z)=\left(\sum_{j=1}^{n}\left(z_{j}-a_{j}\right)^{2}-r^{2}\right)^{-1}
$$

has a pole at $x^{0}$ and is otherwise holomorphic in $\bar{T}_{B}-\left\{x^{0}\right\}$. By taking $r$ slightly larger we achieve that $f(z)$ is still holomorphic at $x^{0}$ but assumes its maximum with respect to $\bar{T}_{B}$ at $x^{0}$.

Hence $x^{0}$ belongs to the Silov boundary of $T_{B} . f\left(z-i y^{0}\right)$, where $y^{0}$ is an arbitrary fixed vector in the $y$-space, takes its maximum at the arbitrary point $z^{0}=x^{0}+i y^{0}$, hence $z^{0}$ belongs to the Silov boundary.

Conversely one sees immediately that if the boundary of $B$ is not strictly convex at $z^{0}$, then $\partial B$ contains a segment of a straight line containing $z^{0}$. Then $T_{B}$ contains a segment of an (at least one-dimensional) analytic surface, for which the maximum principle holds, hence $z^{0}$ does not belong to $S\left(T_{B}\right)$ unless it is an accumulation point of $S\left(T_{B}\right)$. Thus we have proved:

Theorem. Let $T_{B}$ be a convex tube domain. Then the Silov boundary $S\left(T_{B}\right)$ is the closure of the set

$$
\{z \mid x \in S(B), y \text { arbitrary }\}
$$

where $S(B)$ is the closure of the strictly convex boundary points of $B$.

EXAmplEs. If $B$ is a convex polyhedron, then the "corners" are the points where $B$ is strictly convex and hence $S(B)$ consists of them. On the other hand a circle for instance is everywhere strictly convex.

As in the case of pseudo-convex domains we have for arbitrary domains $B$ that are not convex:

$$
S(B) \subseteq \partial B \cap \partial E(B)
$$


where $E(B)$ is the convex envelope of $B$, and correspondingly for the associated tubes

$$
S\left(T_{B}\right) \subseteq \partial T_{B} \cap \partial T_{E(B)}
$$

Finally we should like to note that the characterization of the strictly convex points can be made similarly to that of the strictly pseudo-convex points:

Every convex domain is the limit of domains of the form

$$
B=\{x \mid u(x)<0\},
$$

where $u(x)$ is convex in a neighborhood of $B$.

Each $B$ is convex. Let now $u(x)$ be of $\left(C^{2}\right)$, then one sees easily that a sufficient condition for a point $x^{0}$ to be a strictly convex point is the condition that

$$
\sum \frac{\partial^{2} u(x)}{\partial x_{\mu} \partial x_{\nu}} d x_{\mu} d x_{\nu}>0 .
$$

And if this form is zero for all directions in a neighborhood of a point $x^{0}$, then the boundary of $B$ consists of a hyperplane in a neighborhood of $x^{0}$ and $x^{0}$ is not in $S(B)$. These conditions are exactly analogous to 6.8 .

\section{BiBLIOGRAPHY}

1. R. Arens and M. Singer, Generalized analytic functions, Trans. Amer. Math. Soc. to appear.

2. H. Behnke and K. Stein, Die Konvexität in der Funktionentheorie mehrerer Veränderlichen, Mitteilungen der Math. Gesellschaft in Hamburg, vol. 8 (1940) pp. 34-81.

3. - Die Singularitäten der analytischen Funktionen mehrerer Veränderlichen, Nieuw Arch. Wisk. (1951) pp. 227-242.

4. S. Bergman, Functions of extended class in the theory of functions of several complex variables, Trans. Amer. Math. Soc. vol. 63 (1948) pp. 523-547.

5. - Kernel functions and extended classes in the theory of functions of complex variables, Colloque sur les fonctions de plusieur variables, Bruxèlles (1953) pp. 135-157.

6 . - On zero and pole surfaces of functions of two complex variables, Trans. Amer. Math. Soc. vol. 77 (1954) pp. 413-454.

7. —, Über eine in gewissen Bereichen mit Maximumfläche gültige Integraldarstellung der Funktionen zweier komplexer Variabler I, Math. Z. vol. 39 (1935) pp. 76-94.

8. H. J. Bremermann, Die Charakterisierung von Regularitätsgebieten durch pseudokonvexe Funktionen, Schr. Math. Inst. Univ. Münster, no. 5 (1951).

9. —— Über die Äquivalenz der pseudokonvexen Gebiete und der Holomorphiegebiete im Raum von $n$ komplexen Veränderlichen, Math. Ann. vol. 128 (1954) pp. 63-91.

10. - Complex convexity, Trans. Amer. Math. Soc. vol. 82 (1956) pp. 17-51.

11. - On the conjecture of the equivalence of the plurisubharmonic functions and the Hartogs functions, Math. Ann. vol. 131 (1956) pp. 76-86.

12. - Holomorphic functionals and complex convexity in Banach spaces, Pacific J. Math. vol. 7 (1957) pp. 811-831.

13. - Construction of the envelopes of holomorphy of arbitrary domains, Revista Mat. Hispano-Americana vol. 17 (1957) pp. 1-26. 
14. - Die Charakterisierung Rungescher Gebiete durch plurisubharmonische Funktionen, Math. Ann. vol. 136 (1958) pp. 173-186.

15. C. Carathéodory, On Dirichlet's Problem, Amer. J. Math. vol. 59 (1937) pp. 709-731, Reprinted in: Constantin Carathéodory, Gesammelte mathematische Schriften, vol. III. Munich, 1955.

16. F. Korte, Die Randpunkte der Regularitätsbereiche, Math. Ann. vol. 111 (1935) pp. 119-136.

17. O. D. Kellog, Foundations of potential theory, Berlin, 1929.

18. K. de Leeuw, Functions on circular subsets of the space of $n$ complex variables, Duke Math. J. vol. 24 (1957) pp. 415-431.

19. P. Lelong, Fonctions plurisousharmoniques; mesures de Radon associées. Application aux fonctions analytiques, Colloque sur les fonctions de plusieur variables, Bruxèlles, 1953, pp. 21-40.

20. H. Lewy, On the local character of the solutions of an atypical linear differential equation in three variables and a related theorem for regular functions of two complex variables, Ann. of Math. vol. 64 (1956) pp. 514-522.

21. L. H. Loomis, Abstract harmonic analysis, New York, 1953.

22. D. B. Lowdenslager, Potential theory and a generalized Jensen-Nevanlinna formula for sunctions of several complex variables, J. Math. Mech. vol. 7 (1958) pp. 207-218.

22a. - Potential theory in bounded symmetrical homogeneous complex domains, Ann. Math. vol. 67 (1958) pp. 467-484.

23. H. Mirkil, The work of Šilov on commutative Banach algebras, mimeographed notes, University of Chicago.

24. Martson Morse, Topological methods in the theory of functions of a complex variable, Princeton, 1947.

25. R. Nevanlinna, Eindeutige analytische Funktionen, Berlin, 1953.

26. F. Norguet, Sur les domaines d'holomorphie des fonctions uniformes de plusieur variables complexes. (Passage du local au global), Bull. Soc. Math. France vol. 82 (1954) pp. 137-159.

27. K. Oka, Sur les fonctions analytiques de plusieur variables, IV, Domaines pseudo-convexes, Tôhoku Math. J. vol. 49 (1942) pp. 15-52.

28. - Sur les fonctions analytiques de plusieur variables, IX, Domaines finis sans point critique interieur, Jap. J. Math. vol. 23 (1953) pp. 97-155.

29. O. Perron, Eine neue Behandlung der ersten Randwertaufgabe fibr $\Delta u=0$, Math. Z. vol. 18 (1923) pp. 42-54.

30. G. B. Rizza, Dirichlet problem for n-harmonic functions and related geometrical properties, Math. Ann. vol. 130 (1955) pp. 202-218.

31. F. Severi, Il problema di Dirichlet per le funzioni biarmoniche, Mem. Acc. Italia, vol. 2, no. 1 (1931).

Institute for Advanced Study,

Princeton, N. J. 